\title{
Antithrombotic therapy with intracoronary stenting
}

Steven R Steinhubl, A Michael Lincoff

Following the first report of successful intracoronary stenting in humans, there was early enthusiasm for the potential of this technology to prevent occlusion or restenosis following angioplasty. Such enthusiasm however was quickly tempered by concerns regarding early thrombotic stent occlusion and its associated high risk of myocardial infarction or death, following the report of a $24 \%$ incidence of stent thrombosis in the first 105 patients receiving the Wallstent. ${ }^{1}$ An almost simultaneous report of the first 226 patients receiving a PalmazSchatz stent showed an $18 \%$ rate of stent thrombosis in patients treated with aspirin and dipyridamole, with a dramatic improvement to only $0.6 \%$ following the addition of warfarin. ${ }^{2}$ As management of thrombotic stent occlusion proved to be consistently associated with a poor outcome, a concerted effort was directed towards its prevention through more aggressive antithrombotic regimens. Despite experimentation with a wide variety of regimens, which included combinations of heparin, urokinase, dextran, warfarin, aspirin, dipyridamole, and sulphinpyrazone, stent thrombosis continued to occur in roughly $4 \%$ of patients. Also, these intense antithrombotic protocols contributed to a $10-30 \%$ incidence of vascular complications as well as a substantial prolongation of hospital stays. It was not until after successful stent placement was reported using only antiplatelet therapy in conjunction with intravascular ultrasound guidance to assure optimal stent deployment that the trend towards increasing anticoagulation began to reverse. ${ }^{3}$ Further refinements have now brought us to the point where combined antiplatelet therapy along with high pressure balloon inflations are the standard of care, having produced results that are the basis for the majority of patients receiving stents in many interventional laboratories today.

\section{Pathophysiology of platelets in coronary stenting}

Studies of platelet function in coronary stenting provide a strong physiological basis to target platelet inhibition for the prevention of stent thrombosis. In early studies using a porcine model with intraprocedural dextran and autologous labelled platelets, a rapid increase in platelet deposition at the stent site was demonstrated. ${ }^{4}$ Both this immediate deposition as well as prolonged platelet retention were significantly greater in the stented arteries compared with those treated with balloon angioplasty. These findings were indirectly corroborated in a study comparing platelet activation in patients following balloon angioplasty or stenting. ${ }^{5}$ Subsequent to the implantation of Palmaz-Schatz stents in 30 patients treated with aspirin and heparin followed by phenprocoumon, a significant increase was found in the platelet surface expression of the activated fibrinogen receptor (glycoprotein $\mathrm{IIb} / \mathrm{III}$ ) as well as von Willebrand factor binding and P-selectin expression. These markers of platelet activation peaked at two days following stent placement and coincided with a significant drop in the peripheral platelet count. Importantly, no change was noted in any of these parameters in 30 patients undergoing balloon angioplasty. This same group of investigators then evaluated these markers of platelet activation in stented patients treated either with ticlopidine and aspirin or a standard anticoagulant regimen given at the time of stent placement. ${ }^{6}$ In contrast to the anticoagulant treated patients, those receiving the combined antiplatelet regimen demonstrated platelet deactivation. Interestingly, despite the delayed onset of activity of ticlopidine, a difference was already noted between the antiplatelet and anticoagulant treated patients one day after stent implantation.

The clinical relevance of the platelet surface glycoprotein receptors in stent thrombosis is underlined by the results of a prospective study of haemostatic predictors of stent thrombosis. When glycoprotein IIb/IIIa expression, plasma fibrinogen, and prothrombin fragments $F_{1+2}$ were evaluated in 140 patients undergoing stent implantation followed by anticoagulant therapy, IIb/IIIa expression was found to be the only independent predictor of stent thrombosis. ${ }^{7}$ These findings are important, not only in highlighting the role of platelets in stent thrombosis, but also by demonstrating that the end product of the coagulation cascade, thrombin formation, does not appear to be related to this event.

\section{Clinical application of antiplatelet \\ therapy}

Despite the excessive incidence of stent thrombosis seen during the early experience in patients treated with the antiplatelet combination of aspirin and dipyridamole, a new antiplatelet regimen combining ticlopidine and aspirin began to be re-evaluated when optimal stent expansion using high pressure balloon dilatations was employed. Because data from the initial, non-randomised evalua- 
Table 130 day outcomes in randomised trials of antiplatelet and anticoagulant therapy after coronary artery stenting

\begin{tabular}{|c|c|c|c|}
\hline & $\begin{array}{l}\text { Number } \\
\text { of patients }\end{array}$ & $\begin{array}{l}\text { Primary cardiac } \\
\text { endpoint (\%) }\end{array}$ & $P$ value \\
\hline \multicolumn{4}{|l|}{ ISAR: } \\
\hline $\begin{array}{l}\text { + phenprocoumon } \\
\text { Aspirin + ticlopidine }\end{array}$ & $\begin{array}{l}260 \\
257\end{array}$ & $\begin{array}{l}6 \cdot 2 \% \\
1 \cdot 6 \%\end{array}$ & 0.01 \\
\hline \multicolumn{4}{|l|}{ STARS: } \\
\hline Aspirin + heparin + warfarin & 553 & $2 \cdot 4 \%$ & \\
\hline Aspirin & 555 & $3 \cdot 6 \%$ & $0 \cdot 02^{\star}$ \\
\hline Aspirin + ticlopidine & 544 & $0.6 \%$ & $0.04 \dagger$ \\
\hline \multicolumn{4}{|l|}{ FANTASTIC: } \\
\hline $\begin{array}{l}\text { + oral anticoagulation } \\
\text { Aspirin }+ \text { ticlopidine }\end{array}$ & $\begin{array}{l}230 \\
246\end{array}$ & $\begin{array}{l}4 \cdot 3 \% \\
0 \cdot 8 \%\end{array}$ & 0.03 \\
\hline
\end{tabular}

Primary cardiac endpoint defined as death from cardiac cause, myocardial infarction ( $Q$ wave only in STARS), emergent CABG or re-PTCA

ISAR, Intracoronary Stenting and Antithrombotic Regimen trial'; STARS, Stent Anticoagulation Regimen Study (presented at American Heart Association Scientific Sessions, November 1996); Regimen Study (presented at American Heart Association Scientific Sessions, November 1996);
FANTASTIC, Full Anticoagulation versus Ticlopidine plus Aspirin after Stent Implantation FANTASTIC, Full Anticoagulation versus Ticlopidine plus Aspirin after Stent
(Presented at American Heart Association Scientific Sessions, November 1996).

(Presented at American Heart Association Scientific Sessions, November 1996). ticlopidine plus aspirin versus aspirin alone groups.

tions of this regimen consistently demonstrated subacute stent thrombosis rates of less than $2 \%$ as well as a major reduction in bleeding complications, combined ticlopidine and aspirin quickly became the accepted antiplatelet regimen.

To establish definitely the superiority of antiplatelet rather than anticoagulant therapy following intracoronary stenting, three prospective, randomised trials were designed, which together enrolled over 2600 patients. Although minor variations in design existed between the studies, all compared outcomes in successfully stented patients for a variety of clinical indications, and all employed at least a ticlopidine/aspirin arm and an intravenous heparin/oral vitamin $K$ antagonist/aspirin arm. Each study demonstrated a significant reduction in the primary cardiac end point (death, myocardial infarction, or revascularisation) at 30 days in the combined antiplatelet arm compared with the anticoagulant arm (table 1). In the only published results to date, the Intracoronary Stenting and Antithrombotic Regimen (ISAR) trial reported no haemorrhagic complications in the antiplatelet treated group, compared with a $6.5 \%$ incidence in the anticoagulant arm. ${ }^{8}$ Although the results of these trials have confirmed the beneficial role of antiplatelet compared with anticoagulant treatment in minimising both stent thrombosis and haemorrhagic complications, several issues still remain regarding the optimal antiplatelet strategy.

Despite the encouraging results seen with the combination of ticlopidine and aspirin, concerns over the risk of ticlopidine associated leucopenia, which occurs in up to $2.5 \%$ of patients and is occasionally irreversible, prompted evaluation of whether ticlopidine provided incremental benefit to aspirin alone. Early trials comparing aspirin alone versus aspirin and ticlopidine suggested no difference between the treatment strategies, but these studies involved small numbers of patients and were inadequately powered. The prospective, randomised Stent Anticoagulation Regimen Study (STARS) enrolled 1652 patients and investigated three antithrombotic strategies (table 1); aspirin plus ticlopidine was superior to antithrombotic therapy or aspirin alone (presented at the American Heart Association scientific sessions, November 1996). Interestingly, the aspirin treatment group had the highest incidence of the primary cardiac end point. This outcome is consistent with the results of a porcine carotid stent injury study which suggested a strong synergy between ticlopidine and aspirin in limiting platelet deposition and thrombosis (personal communication, Schwartz RS, 1996).

Although the addition of ticlopidine to aspirin is clearly beneficial, the duration of treatment as well as the optimal time to start therapy has yet to be clearly established. The antiplatelet effects of ticlopidine are dose and time dependent, with near maximal platelet inhibition not achieved until after four days of therapy. Although the results of randomised trials prove that starting ticlopidine at the time of stent placement is adequate for preventing stent thrombosis, preliminary data from our institution suggests that starting ticlopidine several days before the procedure significantly decreases the incidence of asymptomatic but clinically significant post-procedural non-Q wave myocardial infarctions. Also, as stent thrombosis typically occurs within the first week following placement, treatment may not be required for up to four to six weeks as was used in the randomised trials. Limiting the duration of therapy would likely also reduce side effects, as leucopenia secondary to ticlopidine is typically not seen until after two weeks of treatment. Observational data has thus far supported the continued clinical efficacy and improved safety of two weeks of ticlopidine therapy. Further prospective data will be required before the optimal timing of therapy initiation as well as duration of therapy is clearly defined.

\section{Future directions}

The central role of platelet activation in stent thrombosis as well as the proven benefits of antiplatelet therapy suggest a potentially advantageous role for the use of the new class of agents, which potently inhibit the platelet surface membrane glycoprotein IIb/IIIa receptor. Data from small subgroups of patients who received stents for bailout indications in two trials (the Evaluation of PTCA to Improve Long-Term Outcome by c7E3 Glycoprotein Receptor Blockade (EPILOG) and Integrilin to Minimize Platelet Aggregation and Prevent Coronary Thrombosis II (IMPACT II)) support this hypothesis. ${ }^{9}$ Nearly 500 patients in these two studies received stents, and in each study a significant reduction in the 30 day composite cardiac endpoint of death, myocardial infarction and urgent revascularisation, was demonstrated in patients treated with abciximab (Centocor, Malvern, Pennsylvania, USA) or Integrilin (COR Therapeutics, South San Francisco, California, USA), respectively (table 2). The results of two ongoing trials (EPILOG Stent and ERASER) designed to evaluate the use of these agents during 
Table 230 day outcomes with bailout stenting in glycoprotein IIb/IIIa inhibitor trials

\begin{tabular}{|c|c|c|c|c|}
\hline & \multirow{2}{*}{$\begin{array}{l}\text { Total patients } \\
\text { with bailout } \\
\text { stents }\end{array}$} & \multicolumn{2}{|c|}{$\begin{array}{l}\text { Death, } M I \text { or urgent } \\
\text { revascularisation }\end{array}$} & \multirow[b]{2}{*}{ Pvalue } \\
\hline & & IIb/IIIa inhibitor & Placebo & \\
\hline $\begin{array}{l}\text { EPILOG } \\
\text { IMPACT II }\end{array}$ & $\begin{array}{l}325 \\
160\end{array}$ & $\begin{array}{l}9 \cdot 3 \% \\
31 \%\end{array}$ & $\begin{array}{l}22 \cdot 6 \% \\
49 \%\end{array}$ & $\begin{array}{l}0.004 \\
0.021\end{array}$ \\
\hline
\end{tabular}

EPILOG, Evaluation of PTCA to Improve Long-Term Outcome by c7E3 Glycoprotein Receptor Blockade (data on file); IMPACT II, Integrilin to Minimize Platelet Aggregation and Prevent Coronary Thrombosis II. ${ }^{9}$

planned intracoronary stent placement will help clarify their clinical use in all patients receiving stents.

Other areas of ongoing investigation that may impact future antithrombotic drug requirements during stenting include use of less thrombogenic stent materials, local delivery of antithrombotic agents at the stent site, and the use of coated stents. Results with the heparin coated Palmaz-Schatz stent in the Benestent II pilot study were particularly encouraging, in that no episodes of stent thrombosis occurred among 202 patients. $^{10}$

\section{Current technique}

Based on the available data, our present approach for elective intracoronary stent implantation centres around adequate platelet inhibition with the combination of ticlopidine and aspirin. Aspirin is administered at least the evening before and indefinitely thereafter. When possible, ticlopidine is begun at $250 \mathrm{mg}$ twice daily three days before the planned procedure. When ticlopidine is begun just before or at the time of stenting, a $500 \mathrm{mg}$ dose is given, followed by $250 \mathrm{mg}$ twice a day. Twice a day therapy is then typically maintained for two to four weeks. A complete blood count is checked after two and again at four weeks if therapy is maintained. Patients are also instructed to report fevers or other possibly infectious symptoms so that they may be evaluated for neutropenia. Neither warfarin nor low molecular weight heparin are used, and even patients taking chronic anticoagulant therapy are treated with at least two weeks of ticlopidine.

With high risk lesions, the chimeric glycoprotein IIb/IIIa receptor antibody abciximab is frequently employed. Typical indications include stents placed for a bailout indication, lesions with a substantial thrombus burden, culprit lesions in acute coronary syndromes, and saphenous vein grafts (especially degenerated vein grafts). The use of multiple overlapping stents or a suboptimal result after stenting are other situations where abciximab is often used.

\section{Conclusions}

Our knowledge of the physiological response elicited by intracoronary stent placement at both the clinical and molecular level has increased dramatically since the first use of this technology in humans almost 10 years ago. This knowledge has helped us to tailor our technique as well as adjunctive therapies to optimise stenting outcomes. As we continue to explore other avenues for improving coronary stenting, and apply what is learned to clinical practice, stent implantation will approach the ideal as a means to prevent occlusion or restenosis following angioplasty.

1 Serruys PW, Strauss BH, Beatt KJ, Bertrand ME, Puel J, Rickards AF, et al. Angiographic follow-up after placement of a self-expanding coronary-artery stent. $N$ Engl $f$ Med 1991;324:13-17.

2 Schatz RA, Baim DS, Leon M, Ellis SG, Goldberg S, Hirshfeld JW, et al. Clinical experience with the PalmazSchatz coronary stent. Initial results of a multicenter study. Circulation 1991;83:148-61.

3 Colombo A, Hall P, Nakamura S, Almagor Y, Maiello L, Martini G, et al. Intracoronary stenting without anticoagulation accomplished with intravascular ultrasound guidance. Circulation 1995;91:1676-88.

4 Parsson H, Cwikiel W, Johansson K, Swartbol P, Norgren L. Deposition of platelets and neutrophils in porcine iliac Wallstent placement compared with angioplasty alone. Cardiovasc Intervent Radiol

5 Gawaz M, Neumann F-J, Ott I, May A, Rudiger S, Schomig A. Changes in membrane glycoproteins of circulating platelets after coronary stent implantation. Heart 1996;76:166-72.

6 Gawaz M, Neumann F-J, Ott I, May A, Schomig A. Platelet activation and coronary stent implantation. Effect of antithrombotic therapy. Circulation 1996;94: 279-85.

7 Neumann F-J, Gawaz M, Ott I, May A, Mossmer G, Schomig A. Prospective evaluation of hemostatic predictors of subacute stent thrombosis after coronary PalmazSchatz stenting. $¥ \mathrm{Am}$ Coll Cardiol 1996;27:15-21.

8 Schomig A, Neumann F-J, Kastrati A, Schuhlen H, Blasini $R$, Hadamitzky $M$, et al. A randomized comparison of antiplatelet and antiplatelet and anticoagulant therapy after the place1996;334:1084-9.

9 Zidar JP, Kruse KR, Thel MC, Kereiakes D, Muhlestein $\mathrm{JB}$, Davidson CJ, et al. Integrelin for emergency coronary artery stenting. $\mathcal{F}$ Am Coll Cardiol 1996;27:138A.

10 Serruys PW, Emanuelsson $\mathrm{H}$, van der Giessen W, Lunn AC, Kiemeney F, Macaya C, et al. Heparin-coated Palmaz-Schatz stents in human coronary arteries. Early outcome of the Benestent II pilot study. Circulation 1996;93:412-22. 